\title{
Gene expression profile analysis of ENO1 knockdown in gastric cancer cell line MGC-803
}

\author{
ZHIGANG HUANG ${ }^{1,2}$, BODE LIN ${ }^{1}$, HAIYAN PAN ${ }^{1}$, JINLIN DU $^{1}$, RONGWEI HE ${ }^{1}$, \\ SHIZHUO ZHANG ${ }^{1}$ and PING OUYANG ${ }^{3}$ \\ ${ }^{1}$ Department of Epidemiology and Health Statistics, School of Public Health; \\ ${ }^{2}$ Dongguan Key Laboratory of Environmental Medicine; \\ ${ }^{3}$ Guangdong Provincial Key Laboratory of Medical Molecular Diagnostics, Dongguan Scientific Research Center, \\ Guangdong Medical University, Dongguan, Guangdong 523808, P.R. China
}

Received August 8, 2018; Accepted January 25, 2019

DOI: $10.3892 /$ ol.2019.10053

\begin{abstract}
Gastric cancer (GC) is the third leading cause of cancer-associated mortality. In a previous study, we identified that $\alpha$-enolase (ENO1) promoted cell migration in GC, but the underlying molecular mechanisms remain to be fully elucidated. In the present study, small interfering RNAs were identified to interfere with ENO1 expression. The cDNA expression profiling was performed using an Affymetrix mRNA array platform to identify genes that may be associated with ENO1 in human GC cell line MGC-803. The differentially expressed genes (DEGs) were identified using the reverse transcription-quantitative polymerase chain reaction, followed by a series of bioinformatic analyses. As a result, there were 448 DEGs, among which 183 (40.85\%) were downregulated. The most significant functional terms for the DEGs were the nuclear lumen for cell components $\left(\mathrm{P}=2.83 \times 10^{-4}\right)$, transcription for biological processes $\left(\mathrm{P}=3.7 \times 10^{-7}\right)$ and transcription factor activity for molecular functions $\left(\mathrm{P}=1.16 \times 10^{4}\right)$. In total, six significant pathways were enriched, including the most common cancer-associated forkhead box $\mathrm{O}$ signaling pathway
\end{abstract}

Correspondence to: Dr Ping Ouyang, Guangdong Provincial Key Laboratory of Medical Molecular Diagnostics, Dongguan Scientific Research Center, Guangdong Medical University, 1 Xincheng Road, Dongguan, Guangdong 523808, P.R. China

E-mail: ouyangping@gdmu.edu.cn

Abbreviations: AVL9, AVL9 cell migration-associated; DEGs, differentially expressed genes; ENO1, $\alpha$-enolase; FGF2, fibroblast growth factor 2; GC, gastric cancer; GMFB, glia maturation factor $\beta$; GPR180, G-protein-coupled receptor 180; HSPA4, heat shock protein family A, Hsp70 member 4; HUWE1, HECT, UBA and WWE domain-containing 1, E3 ubiquitin protein ligase; MFAP3, microfibrillar-associated protein 3; PPP1CB, protein phosphatase 1 catalytic subunit $\beta$; SEPT8, septin 8; SGK3, serum/glucocorticoidregulated kinase family member 3 ; TPM4, tropomyosin 4

Key words: gastric cancer, $\alpha$-enolase, RNA interference, tumor, microarray
$(\mathrm{P}=0.0077)$, microRNAs in cancer $(\mathrm{P}=0.0183)$ and the cAMP signaling pathway $(\mathrm{P}=0.0415)$. Furthermore, a network analysis identified three hub genes (HUWE1, PPP1CB and HSPA4), which were all involved in tumor metastasis. Taken together, the DEGs, significant pathways and hub genes identified in the present study shed some light on the molecular mechanisms of ENO1 involved in the pathogenesis of GC.

\section{Introduction}

Gastric cancer (GC) is the third leading cause of cancer-related mortality (1-3), with $\sim 1,033,701$ novel diagnoses and 782,685 mortalities worldwide in 2018 (3). Previous studies have indicated that GC may be driven by a number of different genetic abnormalities, such as mutations in cadherin 1 (4) and catenin $\alpha 1$ (5). Chromosomal aberrations including Erb-b2 receptor tyrosine kinase 2 (6), adenomatous polyposis coli, tumor protein p53 and NME/NM23 nucleoside diphosphate kinase 1 (7) have also been frequently identified in GC. Certain SNPs, such as interleukin 17A (rs2275913) (8), mucin 1 (rs4072037) (9) and prostate stem cell antigen (rs2976392) (10), have indicated genetic predispositions towards an increased risk of GC. However, the aforementioned results are not sufficient to clarify the complex pathogenesis of GC. Therefore, further research into the molecular aspects involved in carcinogenesis is required, which will offer new insights into GC treatment.

The $\alpha$-enolase (ENO1) gene encodes a glycolysis-associated enzyme, which contains 434 amino acids and has a molecular mass of $\sim 57 \mathrm{kDa}$ (11). Previous studies have revealed ENO1 to be abnormally expressed in a number of cancer types and serves pivotal roles in tumorigenesis (12-16). For example, in endometrial cancer, ENO1 silencing significantly decreased malignant biological behavior; furthermore, the expression level of ENO1 could affect the prognosis of patients (14). In breast cancer, ENO1 promoted vascular endothelial cell proliferation, inhibited apoptosis and accelerated blood vessel formation (15). In non-small cell lung cancer, stably upregulated ENO1 could activate the focal adhesion kinase/phosphoinositide 3-kinase (PI3K)/protein kinase B pathway and its downstream signals, and then activate 
glycolysis, the cell cycle and epithelial-mesenchymal transition-associated genes $(13,16)$. In colorectal cancer tissues, the expression level of ENO1 was significantly increased, which was associated with tumorigenesis and metastasis in patients with colorectal cancer (17). In addition, an in vitro study suggested that overexpression of ENO1 promoted proliferation, migration and invasion of the colorectal cancer cell line HCT116 (17). However, research regarding the role of ENO1 in GC is insufficient, and further studies are required. To date, only a few studies have indicated that ENO1 can promote chemoresistance in GC, and that increased protein levels of ENO1 lead to a poor prognosis for the patient (18). Previous studies indicated that overexpression of ENO1 can enhance proliferation and migration in GC cell line AGS (19), and that ENO1 can be upregulated by a well-known GC-associated protein, CagA, in AGS cells (20). Combined with the aforementioned results, we hypothesize that ENO1 serves a role in the pathogenesis of GC. Microarray is a powerful tool that can present the whole gene expression profile (21) and, as such, a microarray analysis was performed on ENO1-silencing GC cells with the aim of gaining further understanding into the molecular mechanism(s) of ENO1 in the progression of GC.

\section{Materials and methods}

Cell culture and treatment. The human GC cell line MGC-803 (Sun Yat-sen University Cell Library, Guangdong, China) was cultured as described previously (22). The small fragment small interfering RNA (siRNA) against ENO1 and the scrambled (control) siRNA were synthesized by Beijing Oligobio (Beijing, China). The siRNA-ENO1 sequences were as follows: Forward, 5'-GCAUUGGAGCAGAGGUUU ATT-3' and reverse, 5'-UAAACCUCUGCUCCAAUGCTT-3'. The siRNA transfection experiment was conducted using Lipofectamine ${ }^{\circledR} 2000$ (Invitrogen; Thermo Fisher Scientific Inc., Waltham, MA, USA), according to the manufacturer's protocol. The cells were plated onto 6 -well plates at a density of $8.0 \times 10^{4}$ cells. Following reaching $\sim 50 \%$ confluence, cells were transfected. The cells were assigned to two groups: NC group, transfected with $50 \mathrm{nM}$ scrambled siRNA; and ENO1-knockdown group, transfected with $50 \mathrm{nM}$ siRNA against ENO1. Each group had three parallel samples. Cells were transfected with Lipofectamine 2000 (Thermo Fisher Scientific, Inc., Waltham, MA, USA) in serum- and antibiotic-free Opti-MEM (Thermo Fisher Scientific, Inc.), according to the manufacturer's instructions. After $24 \mathrm{~h}$, the cells were treated with TRIzol ${ }^{\circledR}$ (Thermo Fisher Scientific, Inc.) and the total RNA was extracted.

Microarray analysis. Following extraction of the total RNA from the NC group and ENO1-knockdown group, the quality was determined using NanoDrop ${ }^{\text {TM }}$ 2,000 (Thermo Fisher Scientific, Inc.), and the 2100 Bioanalyzer (Agilent Technologies, Santa Clara, CA, USA). The amplified RNA (aRNA) was prepared using an Affymetrix GeneChip ${ }^{\text {TM }}$ 3'IVT Express kit (Thermo Fisher Scientific, Inc., Waltham, MA, USA), according to the manufacturer's protocol. The aRNA was purified, fragmented and hybridized with the chip probes. Following hybridization, the chip was stained and the final scanned images and raw data were obtained by the Shanghai
GeneChem Co., Ltd. (Shanghai, China). The raw data were processed using the two-way semi-linear model, and the genes with fold change $(\mathrm{FC})>1.5$ and $\mathrm{P}<0.05$ were regarded as significantly DEGs.

Reverse transcription-quantitative polymerase chain reaction (RT-qPCR) analysis. To determine the interference efficiency of siRNA-ENO1 and to validate the gene chip results, ENO1 and five random genes were selected for RT-qPCR analysis and were as follows: AVL9 cell migration-associated (AVL9), glia maturation factor $\beta$ (GFMB), G-protein-coupled receptor 180 (GPR180), microfibrillar-associated protein 3 (MFAP3)and septin 8 (SEPT8). The total RNA was extracted from the cells and the quality was assessed using the aforementioned method. RNA was reverse-transcribed into cDNA using a reverse transcription kit (cat. no. 05091284001; Roche Diagnostics, Basel, Switzerland), according to the manufacturer's protocol. qPCR was carried out in a volume of $10.0 \mu 1$, including 5.0 $\mu \mathrm{l} \mathrm{SYBR}{ }^{\circledR}$ Select Master mix (Roche Diagnostics), $3.4 \mu \mathrm{l}$ DNase/RNase-free water (Beijing Solarbio Science and Technology Co., Ltd., Beijing, China), $1.0 \mu \mathrm{l}$ cDNA, $0.30 \mu \mathrm{l}$ forward primer and $0.30 \mu \mathrm{l}$ reverse primer. $\beta$-actin was selected as the internal reference gene. The Piko Real detection system (Thermo Fisher Scientific, Inc.) was used for the amplification according to the manufacturer's protocol. The primers were synthesized by Generay Biotech Co., Ltd. (Shanghai, China) and the sequences were as follows: ENO1 forward, 5'-GGG AATCCCACTGTTGAGGT-3' and reverse, 5'-CGGAGCTCT AGGGCCTCATA-3'; $\beta$-actin forward, 5'-GGGAAATCGTGC GTGACATTAAGG-3' and reverse, 5'-CAGGAAGGAAGG CTGGAAGAGTG-3'; ALV9 forward, 5'-TTCCATTTCTGG GTGGCAAGT-3' and reverse, 5'-ACATCGTGGTGGTCG GATTTC-3'; GMFB forward, 5'-CAGCGTTGTTCGTTTCTT TGC-3' and reverse, 5'-GTCTTTGGTTGTTTGTGATGT TGC-3'; MFAP3 forward, 5'-AATGACATAGATGCCACC TTG-3' and reverse, 5'-GTGTCCCTCTTCCACCTCTTA-3'; SEPT8 forward, 5'-GGAATAATGTTCACCTTGCTGTCT-3' and reverse, 5'-TTTGCCTCTACTTCATCACGC-3'. For all RT-qPCR experiments, the samples were amplified in triplicate, each consisting of three replicates. The relative levels of target gene mRNA were calculated and normalized relative to $\beta$-actin using the $2^{-\Delta \Delta \mathrm{Cq}}$ method (23).

Functional enrichment analysis. The functional enrichment analyses of the DEGs were performed using DAVID 6.7 (https://david.ncifcrf.gov) (24). Briefly, all the differentially expressed genes (DEGs) were uploaded in the 'Functional Annotation' section of DAVID 6.7, and set $\mathrm{E}=0.01$. The result would indicate the DEGs mapping to different Gene Ontology (GO) terms. The GO annotation (www.geneontology.org) includes three parts: Biological processes (BP), cellular components $(\mathrm{CC})$ and molecular functions (MF), which provide a descriptive framework and functional annotation of DEGs. The pathway enrichment analysis was performed using Kyoto Encyclopedia of Genes and Genomes (KEGG; http://www. genome.jp/kegg) $(25,26) . \mathrm{P}<0.05$ was considered to indicate statistically significant functional terms and pathways.

Protein-protein interaction (PPI) network construction and module selection. A PPI network was constructed based 
on Biological General Repository for Interaction Datasets (BioGRID) in WebGestalt (http://www.webgestalt.org/option. php). The DEGs were mapped to BioGRID and PPI pairs were acquired. Interactions with a confidence score $>0.4$ were retained in the network and were visualized using Cytoscape (version 3.5.1; http://cytoscape.org). In the PPI network, a node represents a protein product of a DEG and the degree represents the number of proteins linked to this node. The nodes with a high degree $(>10)$ were considered to be important and named 'hub genes' in the present study. The PPI modules were screened using the ClusterONE plugin (version 1.0; http://www. paccanarolab.org/clusterone) in Cytoscape (27). Results were considered statistically significant when $\mathrm{P}<0.0005$.

Statistical analysis. All the data were analyzed using SPSS software (version 15.0; SPSS, Inc., Chicago, IL, USA). The measurement data were expressed as the mean \pm standard deviation. Comparison between two groups was performed using an independent sample t-test. $\mathrm{P}<0.05$ was considered to indicate a statistically significant result.

\section{Results}

Successful knockdown of the ENO1 gene in MGC-803 cells. The mRNA expression levels of ENO1 were downregulated 12.08-fold (array data; Fig. 1) and 11.43 \pm 0.39 -fold (RT-qPCR data; Fig. 2) in the ENO1-knockdown group compared with in the NC group. The results indicated that the siRNA fragments targeting the ENO1 gene were successful and that silencing was efficient.

Gene expression profile analysis and hierarchical clustering. The microarray included two groups with six samples, and the heat map results are presented in Fig. 1. As a result, there were 448 DEGs with a FC value $>1.5$ and $\mathrm{P}<0.05$, among which, 183 (40.85\%) were downregulated and 265 (59.15\%) were upregulated. The top ten DEGs with high FC were tropomyosin 4 (TPM4), fibroblast growth factor 2 (FGF2), inhibitor of DNA binding 2, mitochondrial ribosomal protein S33, small integral membrane protein 13, cyclin J, AVL9 cell migration-associated (AVL9), serum/glucocorticoid regulated kinase family member 3 (SGK3), G protein-coupled receptor 180 (GPR180) and mesoderm development LRP chaperone.

Verification of the array data using $R T-q P C R$ analysis. Five DEGs (AVL9, GMFB, GPR180, MFAP3 and SEPT8) were selected for qPCR analysis. The results (Fig. 2) indicated that the mRNA levels of AVL9, GMFB, GPR180, MFAP3 and SEPT8 were downregulated 2.78-, 2.68-, 2.72-, 2.67- and 2.23-fold, respectively. In the RT-qPCR experiment, these genes were downregulated 3.05 $\pm 0.07-, 2.33 \pm 0.12-, 3.15 \pm 0.06-$, $2.77 \pm 0.08$ - and $2.89 \pm 0.12$-fold, respectively. The array data were in concordance with the RT-qPCR results.

Functional annotation analysis of the DEGs. The gene annotation analysis was performed using DAVID and the detailed results (Table I) identify the number of significant functional classifications for BP, CC and MF as 26, 7 and 10, respectively. The DEGs were mainly enriched in transcription, blood vessel morphogenesis and cell cycle for BP. CC enrichment was detected for genes associated with the nuclear lumen, organelle lumen and nucleoplasm, and MF enrichment was identified for genes associated with transcription factor activity, transcription regulator activity and cytoskeletal protein binding.

KEGG pathway enrichment analysis. The DEGs responding to ENO1 silencing were enriched in six significant pathways: Systemic lupus erythematosus [Homo sapiens (hsa)05322; $\mathrm{P}<0.001$ ], viral carcinogenesis (hsa05203; $\mathrm{P}=0.00141$ ), alcoholism (hsa05034; $\mathrm{P}=0.003$ ), forkhead box O (FoxO) signaling pathway (hsa04068; $\mathrm{P}=0.0077$ ), miRNAs in cancer (hsa05206; $\mathrm{P}=0.0183$ ) and cAMP signaling pathway (hsa04024; P=0.0415) (Table II).

PPI network analysis. The PPIs among the 448 DEGs were predicted using WebGestalt with information from BioGRID. The constructed network consisted of 209 proteins (nodes) and 293 interactions (edges) (Fig. 3). In addition, there were seven genes that had high degrees with edges $\geq 10$ in the PPI network. These seven genes were HECT, UBA and WWE domain-containing 1, E3 ubiquitin protein ligase (HUWE1; degree, 16), protein phosphatase 1 catalytic subunit $\beta$ (PPP1CB; degree, 16), heat shock protein family A (Hsp70) member 4 (HSPA4; degree, 16), signal transducer and activator of transcription 3 (STAT3; degree, 13), anillin actin-binding protein (degree, 12), Src homology 3 domain-containing kinase-binding protein 1 (degree, 10) and casein kinase $2 \alpha 2$ (degree, 10), respectively. Among these, HUWE1, PPP1CB and HSPA4 were the top three nodes with 16 edges.

Module analysis and protein domain analysis. ClusterONE was applied for module analysis to further predict potential protein complexes. For the network constructed above, there were three significant modules ( $\mathrm{P}<0.0005$; Fig. 4$)$, as follows: Module A (nodes, 24; density, 0.101; quality, 0.549; $\mathrm{P}=0.00000791$ ); Module B (Nodes, 18; density, 0.183; quality, 0.683; $\mathrm{P}=0.000136$ ) and Module $\mathrm{C}$ (nodes, 17; density, 0.132; quality, $0.581 ; \mathrm{P}=0.00045$ ) (Table III). For the protein domain analysis, one significant domain was found for Module B: Myosin head, motor domain (IPR001609) ( $\mathrm{P}=0.034)$. No significantly enriched protein domains were identified in Modules A and C.

\section{Discussion}

In the present study, a total of 448 DEGs responded to ENO1 knockdown in the human GC cell line MGC-803. Certain DEGs that demonstrated significantly decreased expression in the present study, including TPM4, have been reported to be associated with clinical progression in patients with colon cancer (28). Another gene, SGK3, which was identified to be downregulated in the present study has previously been reported to serve an important role in the development of breast cancer (29). In addition, FGF2, which also demonstrated significantly decreased expression, is a well-known oncogene $(30,31)$.

The DEGs were enriched in six significant pathways: Systemic lupus erythematosus, viral carcinogenesis, alcoholism, FoxO signaling pathway, miRNAs in cancer and 
Table I. GO analysis of the DEGs regulated by ENO1 silencing.

\begin{tabular}{|c|c|c|c|}
\hline Identifier & Functional term & Count & P-value \\
\hline \multicolumn{4}{|c|}{ Biological process } \\
\hline GO:0006350 & Transcription & 86 & $6.43 \times 10^{-7}$ \\
\hline GO:0006355 & Regulation of transcription, DNA-dependent & 73 & $5.37 \times 10^{-6}$ \\
\hline GO:0045449 & Regulation of transcription & 96 & $1.08 \times 10^{-5}$ \\
\hline GO:0051252 & Regulation of RNA metabolic process & 73 & $1.18 \times 10^{-5}$ \\
\hline GO:0010629 & Negative regulation of gene expression & 28 & $1.00 \times 10^{-4}$ \\
\hline GO:0016481 & Negative regulation of transcription & 26 & $1.40 \times 10^{-4}$ \\
\hline GO:0006357 & Regulation of transcription from RNA polymerase II promoter & 35 & $1.81 \times 10^{-4}$ \\
\hline GO:0048514 & Blood vessel morphogenesis & 16 & $1.98 \times 10^{-4}$ \\
\hline GO:0010605 & Negative regulation of macromolecule metabolic process & 35 & $2.17 \times 10^{-4}$ \\
\hline GO:0045934 & $\begin{array}{l}\text { Negative regulation of nucleobase, nucleoside, nucleotide and nucleic } \\
\text { acid metabolic process }\end{array}$ & 27 & $3.13 \times 10^{-4}$ \\
\hline GO:0010558 & Negative regulation of macromolecule biosynthetic process & 28 & $3.74 \times 10^{-4}$ \\
\hline GO:0051172 & Negative regulation of nitrogen compound metabolic process & 27 & $3.82 \times 10^{-4}$ \\
\hline GO:0045892 & Negative regulation of transcription, DNA-dependent & 21 & $4.39 \times 10^{-4}$ \\
\hline GO:0051253 & Negative regulation of RNA metabolic process & 21 & $5.34 \times 10^{-4}$ \\
\hline GO:0031327 & Negative regulation of cellular biosynthetic process & 28 & $5.50 \times 10^{-4}$ \\
\hline GO:0009890 & Negative regulation of biosynthetic process & 28 & $7.64 \times 10^{-4}$ \\
\hline GO:0001568 & Blood vessel development & 16 & $9.55 \times 10^{-4}$ \\
\hline GO:0001525 & Angiogenesis & 12 & $9.60 \times 10^{-4}$ \\
\hline GO:0007049 & Cell cycle & 34 & $1.189962 \times 10^{-3}$ \\
\hline GO:0001944 & Vasculature development & 16 & $1.215671 \times 10^{-3}$ \\
\hline GO:0031099 & Regeneration & 8 & $1.374438 \times 10^{-3}$ \\
\hline GO:0051726 & Regulation of cell cycle & 18 & $3.03174 \times 10^{-3}$ \\
\hline GO:0031100 & Organ regeneration & 5 & $3.326962 \times 10^{-3}$ \\
\hline GO:0007507 & Heart development & 13 & $6.280962 \times 10^{-3}$ \\
\hline GO:0030593 & Neutrophil chemotaxis & 4 & $8.795164 \times 10^{-3}$ \\
\hline GO:0007167 & Enzyme-linked receptor protein signaling pathway & 17 & $9.52888 \times 10^{-3}$ \\
\hline \multicolumn{4}{|l|}{ Cell component } \\
\hline GO:0031981 & Nuclear lumen & 52 & $2.58 \times 10^{-4}$ \\
\hline GO:0070013 & Intracellular organelle lumen & 60 & $3.75 \times 10^{-4}$ \\
\hline GO:0043233 & Organelle lumen & 60 & $6.74 \times 10^{-4}$ \\
\hline GO:0031974 & Membrane-enclosed lumen & 60 & $1.097428 \times 10^{-3}$ \\
\hline GO:0005654 & Nucleoplasm & 34 & $1.312471 \times 10^{-3}$ \\
\hline GO:0005783 & Endoplasmic reticulum & 34 & $4.951755 \times 10^{-3}$ \\
\hline GO:0044451 & Nucleoplasm part & 22 & $8.735324 \times 10^{-3}$ \\
\hline \multicolumn{4}{|c|}{ Molecular function } \\
\hline GO:0003700 & Transcription factor activity & 43 & $2.18 \times 10^{-4}$ \\
\hline GO:0030528 & Transcription regulator activity & 58 & $5.22 \times 10^{-4}$ \\
\hline GO:0008092 & Cytoskeletal protein binding & 26 & $6.25 \times 10^{-4}$ \\
\hline GO:0016564 & Transcription repressor activity & 18 & $1.995073 \times 10^{-3}$ \\
\hline GO:0043014 & $\alpha$-tubulin binding & 4 & $2.056678 \times 10^{-3}$ \\
\hline GO:0003677 & DNA binding & 77 & $3.813995 \times 10^{-3}$ \\
\hline GO:0046914 & Transition metal ion binding & 88 & $6.180415 \times 10^{-3}$ \\
\hline GO:0003779 & Actin binding & 17 & $6.453361 \times 10^{-3}$ \\
\hline GO:0008270 & Zinc ion binding & 75 & $6.826083 \times 10^{-3}$ \\
\hline GO:0004672 & Protein kinase activity & 26 & $7.297114 \times 10^{-3}$ \\
\hline
\end{tabular}

GO, Gene Ontology. 
Table II. Detailed information of the Kyoto Encyclopedia of Genes and Genomes pathway analysis.

\begin{tabular}{clcll}
\hline Identifier & \multicolumn{1}{c}{ Name } & Count & \multicolumn{1}{c}{ Gene } & P-value \\
\hline hsa05322 & Systemic lupus erythematosus & 6 & $\begin{array}{l}\text { HIST1H2BD, HIST1H2BG, HIST1H2BF, HIST1H2BE, } \\
\text { HIST1H2BI, HIST1H2BC }\end{array}$ & $<0.001$ \\
hsa05203 & Viral carcinogenesis & 10 & $\begin{array}{l}\text { CDKN1A, HIST1H2BD, CCND1, STAT3, TRAF3, } \\
\text { HIST1H2BG, HIST1H2BF, HIST1H2BE, HIST1H2BI, }\end{array}$ & $1.41 \times 10^{-3}$ \\
hsa05034 & Alcoholism & 6 & $\begin{array}{l}\text { HIST1H2BC } \\
\text { HIST1H2BD, HIST1H2BG, HIST1H2BF, HIST1H2BE, }\end{array}$ & $3.11 \times 10^{-3}$ \\
hsa04068 & FoxO signaling pathway & 6 & $\begin{array}{l}\text { HIST1H2BI, HIST1H2BC } \\
\text { CDKN1A, SGK3, CCND1, STAT3, TGFBR1, SETD7 }\end{array}$ & $7.7 \times 10^{-3}$ \\
hsa05206 & MicroRNAs in cancer & 8 & $\begin{array}{l}\text { CDKN1A, CYP1B1, DICER1, PDCD4, MCL1, PLAU, } \\
\text { CCND1, STAT3 }\end{array}$ & $1.83 \times 10^{-2}$ \\
hsa04024 & cAMP signaling pathway & 5 & ADCY9, HTR1D, PPP1CB, RAC1, CREB3L2 & $4.15 \times 10^{-2}$ \\
\hline
\end{tabular}

hsa, Homo sapiens; FoxO, Forkhead box O; cAMP, cyclic adenosine monophosphate.

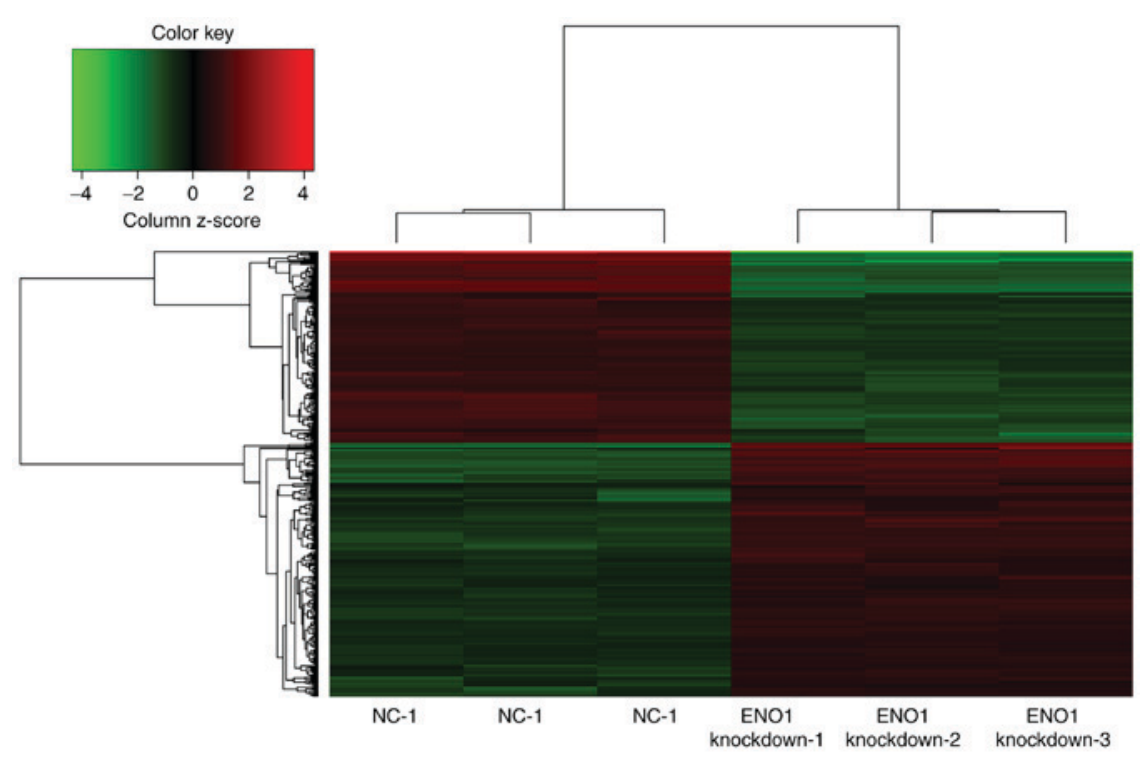

Figure 1. Array data. Hierarchical clustering dendrogram comparing MGC-803 cells transfected with scrambled siRNA (control) and siRNA against ENO1. Each sample represents the average gene expression value for three replicates. Green, low expression; red, high expression. siRNA, small interfering RNA; NC, negative control; ENO1 knockdown, siRNA against ENO1; ENO1, $\alpha$-enolase.

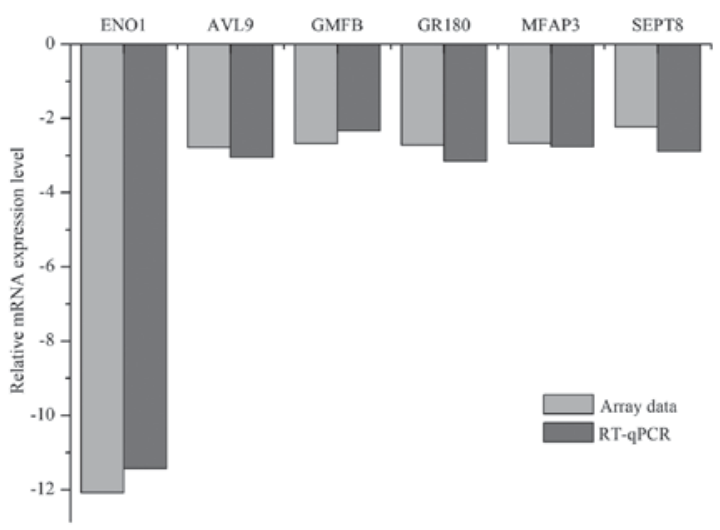

Figure 2. RT-qPCR verification of the array data. Fold changes of selected genes in NC and ENO1-knockdown groups using microarray and RT-qPCR analyses. RT-qPCR, reverse transcription-quantitative polymerase chain reaction; ENO1, $\alpha$-enolase; AVL9, AVL9 cell migration-associated; GMFB, glia maturation factor $\beta$; GPR180, G-protein-coupled receptor 180; MFAP3, microfibrillar-associated protein 3 ; SEPT8, septin 8 . All values are relative to the negative controls.
cAMP signaling pathway (Table II). Among these, some were reported to be associated with cancer development, such as the cAMP signaling pathway and FoxO signaling pathway. cAMP signaling regulates protein levels by controlling gene transcription via transcriptional activators that are involved in cancer cell migration, proliferation, apoptosis, and cytoskeleton remodeling in bladder cancer (32), breast cancer (33) and lung cancer (34). Furthermore, an exchange protein directly activated by cAMP (EPAC1) has been regarded as a prognostic marker and may be a potential therapy target for GC (35), which suggests the important role that the cAMP signaling pathway serves in the pathological processes of GC. The FoxO signaling pathway has also been reported to be associated with breast cancer (36), bladder cancer (37), prostate cancer (38) and lung cancer (39). Previous studies have demonstrated that there may be crosstalk between the cAMP signaling pathway and the FoxO signaling pathway. On one hand, activation of the cAMP signaling pathway increased FoxO1 phosphorylation (40). On 


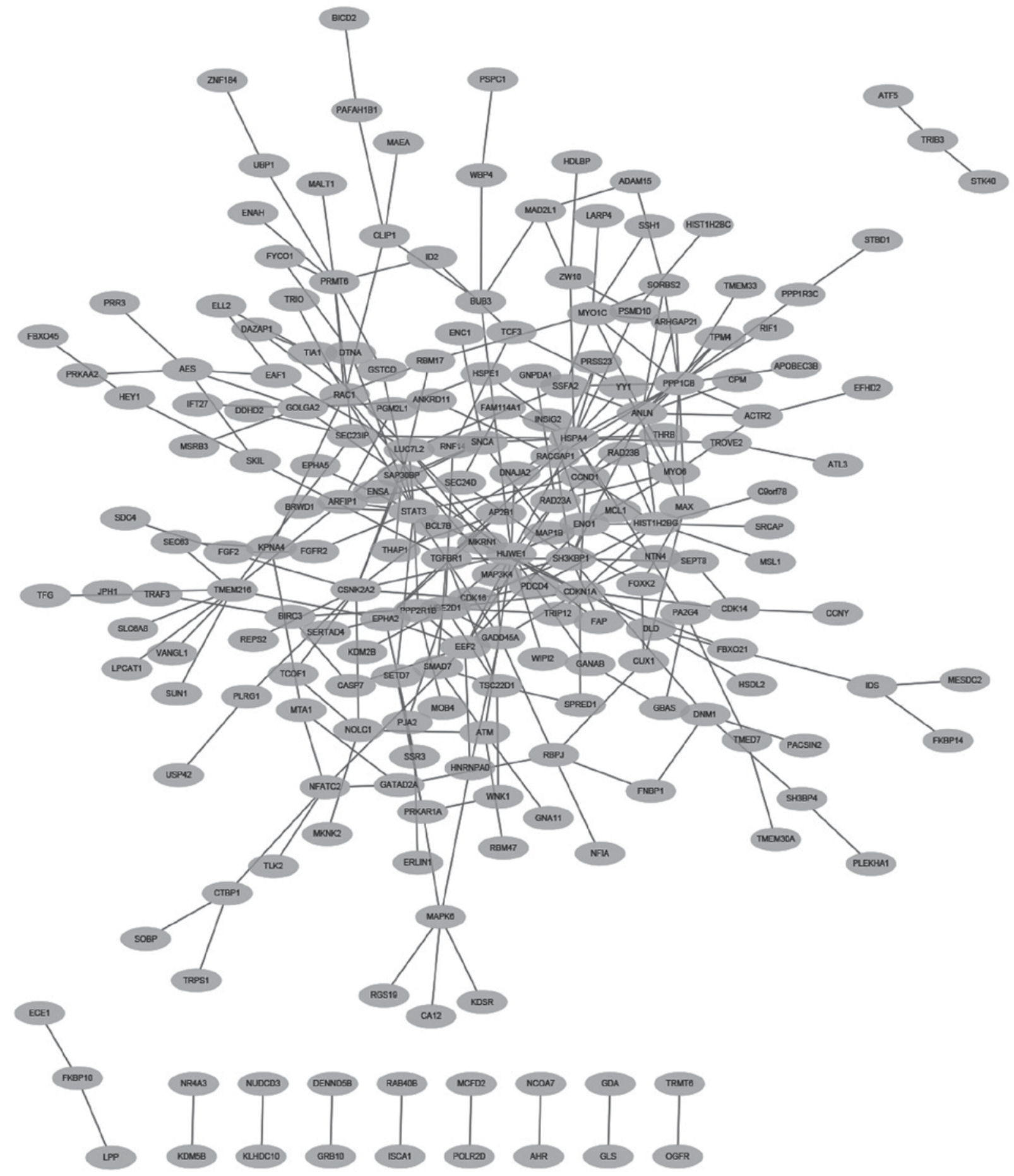

Figure 3. Protein-protein interaction (PPI) network of differentially expressed genes (DEGs) based on BioGRID. The network contained 209 nodes and 293 edges. There were seven genes with a high degree $\geq 10$ and the first three, HUWE1, PPP1CB and HSPA4, all had a degree of 16. HUWE1, HECT, UBA and WWE domain-containing 1, E3 ubiquitin protein ligase; PPP1CB, protein phosphatase 1 catalytic subunit $\beta$; HSPA4, heat shock protein family A (Hsp70) member 4.

the other hand, FoxOs supported the metabolic requirements of normal and tumor cells via the PI3K signaling pathway, which was reported to interact with the cAMP signaling pathway in a number of physiological processes (41). In addition, systemic lupus erythematosus was the most significant pathway response to ENO1 inhibition, and this result was similar to that in our previous study on TPI silencing (22). As previously discussed, although no definitive evidence has suggested the involvement of systemic lupus erythematosus in the pathogenesis of GC, there may be an association between the two since certain sporadic patients were affected by GC and systemic lupus erythematosus simultaneously $(22,42,43)$. The molecular mechanisms underlying the involvement of systemic lupus erythematosus in the pathogenesis of GC are not currently fully understood and further research is required.

The constructed PPI network based on BioGRID included 209 nodes and 293 edges. There were seven DEGs with a degree $\geq 10$, among which, the first three were PPP1CB, HUWE1 and HSPA4, which were regarded as hub genes and may interact with ENO1 in GC progression. These 
Table III. Detailed information of three modules screened from the PPI network of all DEGs.

\begin{tabular}{|c|c|c|c|c|c|}
\hline Module & Nodes & Density & Quality & P-value & Members \\
\hline A & 24 & 0.101 & 0.549 & $7.905 \times 10^{-6}$ & $\begin{array}{l}\text { HSPE1, PGM2L1, HSPA4, ARFIP1, TMED7, TMEM30A, } \\
\text { HUWE1, GANAB, GBAS, TROVE2, ATL3, SEC24D, } \\
\text { FAM114A1, ZW10, HDLBP, FAP, PA2G4, DNAJA2, WIPI2, } \\
\text { MCL1, PRSS23, THRB, PSMD10, HIST1H2BC }\end{array}$ \\
\hline B & 18 & 0.183 & 0.683 & $1.356 \times 10^{-4}$ & $\begin{array}{l}\text { SORBS2, ARHGAP21, ANLN, PPP1CB, TPM4, SSFA2, } \\
\text { MYO1C, LARP4, SSH1, ACTR2, EFHD2, STBD1, PPP1R3C, } \\
\text { MYO6, CPM, RIF1, APOBEC3B, TMEM33 }\end{array}$ \\
\hline $\mathrm{C}$ & 17 & 0.132 & 0.581 & $4.502 \times 10^{-4}$ & $\begin{array}{l}\text { RGS19, MAPK6, CUX1, FOXK2, NFATC2, TLK2, MAP1B, } \\
\text { GATAD2A, RBPJ, MTA1, HNRNPA0, NFIA, KDSR, CA12, } \\
\text { MAX, GADD45A, FNBP1 }\end{array}$ \\
\hline
\end{tabular}

A

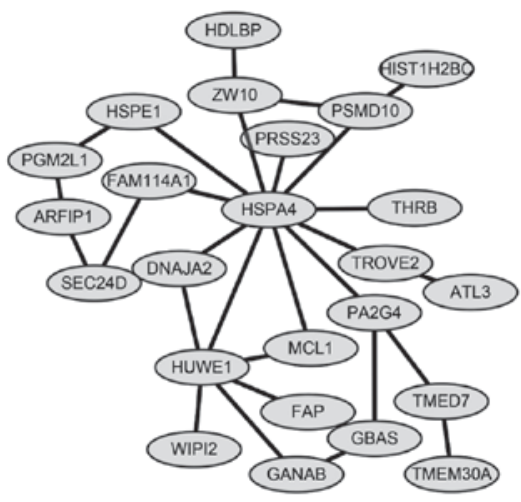

B

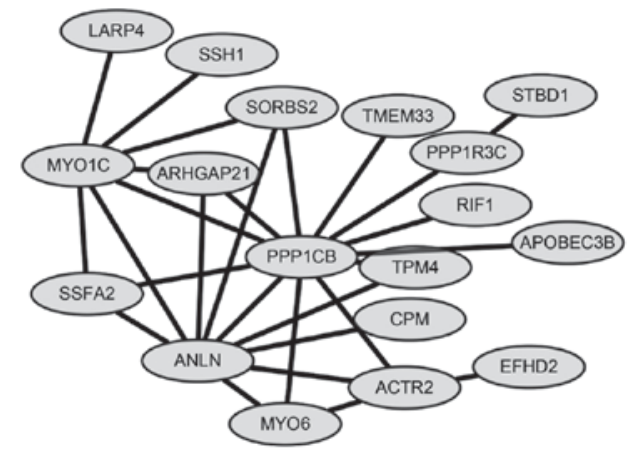

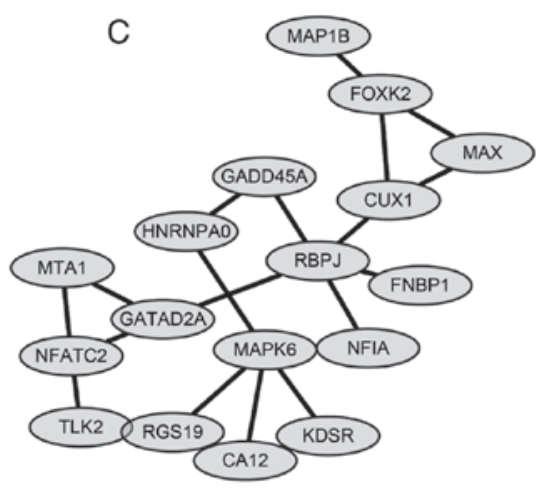

Figure 4. Modules identified from the network based on BioGRID. Three modules were identified with $\mathrm{P}<5 \times 10^{-4}$. Module A (nodes, 24; density, 0.101; quality, $0.549 ; \mathrm{P}=7.91 \times 10^{-6}$ ); Module $\mathrm{B}$ (nodes, 18 ; density, 0.183 ; quality, $0.683 ; \mathrm{P}=1.36 \times 10^{-4}$ ) and Module $\mathrm{C}$ (nodes, 17 ; density, $0.132 ;$ quality, $0.581 ; \mathrm{P}=4.50 \times 10^{-4}$ ).

three genes have been reported to be associated with cancer development. PPP1CB, encoding protein phosphatase 1 catalytic subunit $\beta$ isoform, has been reported in prostate cancer, chronic lymphocytic leukemia (44) and even used as a potential biomarker for distinguishing malignant melanoma from other melanocytic lesions (45). HUWE1, as a ubiquitin ligase, has been regarded as a tumor suppressor and served key roles in tumorigenesis (46). For example, compared with normal thyroid tissue, HUWE1 was downregulated in human thyroid cancer tissues, and overexpression of HUWE1 in thyroid cancer cells enhanced chemotherapeutic sensitivity and inhibition of cell proliferation, cell migration and invasion (47). The third hub gene HSPA4 encodes a heat shock 70 protein. It has been reported that upregulation of heat shock proteins (HSPA12A, HSP90B1, HSPA4, HSPA5 and HSPA6) in tumor tissues is associated with poor outcomes from hepatitis B virus-associated early-stage hepatocellular carcinoma (48). Furthermore, HSPA4 has been reported to regulate cell migration and delay gastric ulcer healing (49). To the best of our knowledge, the present study is the first to identify the possible association between PPP1CB, HUWE1, HSPA4 and ENO1. Further studies are required to confirm these connections and their functions in the pathogenesis in $\mathrm{GC}$ tumorigenesis.

In summary, the results of the present study provide a comprehensive bioinformatic analysis of the genes associated with ENO1. The important signaling pathways (such as cAMP signaling pathway and FoxO signaling pathway) and key genes 
(such as PPP1CB, HUWE1 and HSPA4) may help to narrow down the role of ENO1 in the pathogenesis and treatment of GC.

\section{Acknowledgements}

The authors would like to thank, Dr Tao Li, Dr Yuqing He, Dr Zunnan Huang, Dr Jindong Ni and Dr Huanwen Tang of Guangdong Medical University, for their advice on the present study.

\section{Funding}

The present study was supported by grants from the Natural Science Fund Project of Guangdong Province, China (no. 2016A030313683), the Social Science and Technology Development Project of Dongguan, Guangdong Province, China (nos. 2014108101051 and 2016108101039) and the National Natural Science Foundation of China (no. 81200082).

\section{Availability of data and materials}

The datasets generated and/or analyzed during the study are available from the corresponding author upon request.

\section{Authors' contributions}

$\mathrm{ZH}$ and $\mathrm{PO}$ designed the experiments, performed the bioinformatic analysis and wrote the paper; BL and HP identified the correct siRNA fragment and performed the array; JD carried out the statistical analysis; RH performed the RT-qPCR experiment; SZ cultured the cells.

\section{Ethics approval and consent to participate}

Not applicable.

\section{Patient consent for publication}

Not applicable.

\section{Competing interests}

The authors declare that they have no competing interests.

\section{References}

1. Massimo R, Fassan M and Graham DY: Epidemiology of gastric cancer. In: Gastric Cancer. Springer, Switzerland, pp23-34, 2015.

2. Torre LA, Bray F, Siegel RL, Ferlay J, Lortet-Tieulent J and Jemal A: Global cancer statistics, 2012. CA Cancer J Clin 65 87-108, 2015

3. Bray F, Ferlay J, Soerjomataram I, Siegel RL, Torre LA and Jemal A: Global Cancer Statistics 2018: GLOBOCAN estimates of incidence and mortality worldwide for 36 Cancers in 185 Countries. CA Cancer J Clin 68: 394-424, 2018.

4. van Roy F: Beyond E-cadherin: Roles of other cadherin superfamily members in cancer. Nat Rev Cancer 14: 121-134, 2014.

5. Majewski IJ, Kluijt I, Cats A, Scerri TS, de Jong D, Kluin RJ, Hansford S, Hogervorst FB, Bosma AJ, Hofland I, et al: An alpha-E-catenin (CTNNA1) mutation in hereditary diffuse gastric cancer. J Pathol 229: 621-629, 2013.

6. Pazo Cid RA and Anton A: Advanced HER2-positive gastric cancer: Current and future targeted therapies. Crit Rev Oncol Hematol 85: 350-362, 2013
7. Gazvoda B, Juvan R, Zupanic-Pajnic I, Repse S, Ferlan-Marolt K, Balazic J and Komel R: Genetic changes in Slovenian patients with gastric adenocarcinoma evaluated in terms of microsatellite DNA. Eur J Gastroenterol Hepatol 19: 1082-1089, 2007.

8. Zhang X, Zheng L, Sun Y and Zhang X: Analysis of the association of interleukin-17 gene polymorphisms with gastric cancer risk and interaction with Helicobacter pylori infection in a Chinese population. Tumour Biol 35: 1575-1580, 2014.

9. Saeki N, Saito A, Choi IJ, Matsuo K, Ohnami S, Totsuka H, Chiku S, Kuchiba A, Lee YS, Yoon KA, et al: A functional single nucleotide polymorphism in mucin 1 , at chromosome 1q22, determines susceptibility to diffuse-type gastric cancer. Gastroenterology 140: 892-902, 2011.

10. Garcia-Gonzalez MA, Bujanda L, Quintero E, Santolaria S, Benito R, Strunk M, Sopeña F, Thomson C, Pérez-Aisa A, Nicolás-Pérez D, et al: Association of PSCA rs2294008 gene variants with poor prognosis and increased susceptibility to gastric cancer and decreased risk of duodenal ulcer disease. Int J Cancer 137: 1362-1373, 2015.

11. Zakrzewicz D, Didiasova M, Zakrzewicz A, Hocke AC, Uhle F, Markart P, Preissner KT and Wygrecka M: The interaction of enolase-1 with caveolae-associated proteins regulates its subcellular localization. Biochem J 460: 295-307, 2014.

12. Lee SY, Jin CC, Choi JE, Hong MJ, Jung DK, Do SK, Baek SA, Kang HJ, Kang HG, Choi SH, et al: Genetic polymorphisms in glycolytic pathway are associated with the prognosis of patients with early stage non-small cell lung cancer. Sci Rep 6: 35603, 2016.

13. Dai L, Qu Y, Li J, Wang X, Wang K, Wang P, Jiang BH and Zhang J: Serological proteome analysis approach-based identification of ENO1 as a tumor-associated antigen and its autoantibody could enhance the sensitivity of CEA and CYFRA 21-1 in the detection of non-small cell lung cancer. Oncotarget 8: 36664-36673, 2017.

14. Zhao M, Fang W, Wang Y, Guo S, Shu L, Wang L, Chen Y, Fu Q, Liu Y, Hua S, et al: Enolase-1 is a therapeutic target in endometrial carcinoma. Oncotarget 6: 15610-15627, 2015.

15. Gao J, Zhao R, Xue Y, Niu Z, Cui K, Yu F, Zhang B and Li S: Role of enolase-1 in response to hypoxia in breast cancer: Exploring the mechanisms of action. Oncol Rep 29: 1322-1332, 2013.

16. Fu QF, Liu Y, Fan Y, Hua SN, Qu HY, Dong SW, Li RL, Zhao MY, Zhen Y, Yu XL, et al: Alpha-enolase promotes cell glycolysis, growth, migration, and invasion in non-small cell lung cancer through FAK-mediated PI3K/AKT pathway. J Hematol Oncol 8: $22,2015$.

17. Zhan P, Zhao S, Yan H, Yin C, Xiao Y, Wang Y, Ni R, Chen W, Wei $\mathrm{G}$ and Zhang P: $\alpha$-enolase promotes tumorigenesis and metastasis via regulating AMPK/mTOR pathway in colorectal cancer. Mol Carcinog 56: 1427-1437, 2017.

18. Qian X, Xu W, Xu J, Shi Q, Li J, Weng Y, Jiang Z, Feng L, Wang X, Zhou J and Jin H: Enolase 1 stimulates glycolysis to promote chemoresistance in gastric cancer. Oncotarget 8: 47691-47708, 2017.

19. Liu YQ, Huang ZG, Li GN, Du JL, Ou YP, Zhang XN, Chen TT and Liang QL: Effects of $\alpha$-enolase (ENO1) over-expression on malignant biological behaviors of AGS cells. Int J Clin Exp Med 8: 231-239, 2015.

20. Chen S, Duan G, Zhang R and Fan Q: Helicobacter pylori cytotoxin-associated gene A protein upregulates $\alpha$-enolase expression via Src/MEK/ERK pathway: implication for progression of gastric cancer. Int J Oncol 45: 764-770, 2014.

21. Qin Y and Tian YP: A microarray gene analysis of peripheral whole blood in normal adult male rats after long-term GH gene therapy. Cell Mol Biol Lett 15: 177-195, 2010.

22. Ouyang P, Lin B, Du J, Pan H, Yu H, He R and Huang Z: Global gene expression analysis of knockdown Triosephosphate isomerase (TPI) gene in human gastric cancer cell line MGC-803. Gene 647: 61-72, 2018.

23. Livak KJ and Schmittgen TD: Analysis of relative gene expression data using real-time quantitative PCR and the 2(-Delta Delta C(T)) method. Methods 25: 402-408, 2001.

24. Wang Q: Identification of biomarkers for metastatic osteosarcoma based on DNA microarray data. Neoplasma 62: 365-371, 2015.

25. Li HY, Jin N, Han YP and Jin XF: Pathway crosstalk analysis in prostate cancer based on protein-protein network data. Neoplasma 64: 22-31, 2017.

26. Wang Y, Jiang T, Li Z, Lu L, Zhang R, Zhang D, Wang X and Tan J: Analysis of differentially co-expressed genes based on microarray data of hepatocellular carcinoma. Neoplasma 64: 216-221, 2017. 
27. Li M, Li D, Tang Y, Wu F and Wang J: CytoCluster: A cytoscape plugin for cluster analysis and visualization of biological networks. Int J Mol Sci 18: pii: E1880, 2017.

28. Yang R, Zheng G, Ren D, Chen C, Zeng C, Lu W and Li H: The clinical significance and biological function of tropomyosin 4 in colon cancer. Biomed Pharmacother 101: 1-7, 2018.

29. Sun X, Liu X, Liu BO, Li S, Zhang D and Guo H: Serum- and glucocorticoid-regulated protein kinase 3 overexpression promotes tumor development and aggression in breast cancer cells. Oncol Lett 12: 437-444, 2016.

30. Coleman SJ, Chioni AM, Ghallab M, Anderson RK, Lemoine NR, Kocher HM and Grose RP: Nuclear translocation of FGFR1 and FGF2 in pancreatic stellate cells facilitates pancreatic cancer cell invasion. EMBO Mol Med 6: 467-481, 2014.

31. Xu M, Gu M, Zhang K, Zhou J, Wang Z and Da J: miR-203 inhibition of renal cancer cell proliferation, migration and invasion by targeting of FGF2. Diagn Pathol 10: 24, 2015.

32. Ichikawa $H$, Itsumi M, Kajioka S, Maki T, Lee K, Tomita M and Yamaoka S: Overexpression of exchange protein directly activated by cAMP-1 (EPAC1) attenuates bladder cancer cell migration. Biochem Biophys Res Commun 495: 64-70, 2018.

33. Kumar N, Gupta S, Dabral S, Singh S and Sehrawat S: Role of exchange protein directly activated by cAMP (EPAC1) in breast cancer cell migration and apoptosis. Mol Cell Biochem 430: 115-125, 2017.

34. Park JY and Juhnn YS: cAMP signaling increases histone deacetylase 8 expression via the Epac2-Rap1 A-Akt pathway in H1299 lung cancer cells. Exp Mol Med 49: e297, 2017.

35. Sun DP, Fang CL, Chen HK, Wen KS, Hseu YC, Hung ST, Uen $\mathrm{YH}$ and Lin KY: EPAC1 overexpression is a prognostic marker and its inhibition shows promising therapeutic potential for gastric cancer. Oncol Rep 37: 1953-1960, 2017.

36. Bullock M: FOXO factors and breast cancer: Outfoxing endocrine resistance. Endocr Relat Cancer 23: R113-130, 2016.

37. Tang M, Zhao Y, Liu N, Chen E, Quan Z, Wu X and Luo C: Overexpression of HepaCAM inhibits bladder cancer cell proliferation and viability through the $\mathrm{AKT} / \mathrm{FoxO}$ pathway. J Cancer Res Clin Oncol 143: 793-805, 2017.

38. Yang Y, Blee AM, Wang D, An J, Pan Y, Yan Y, Ma T, He Y, Dugdale J, Hou X, et al: Loss of FOXO1 cooperates with TMPRSS2-ERG overexpression to promote prostate tumorigenesis and cell invasion. Cancer Res 77: 6524-6537, 2017.

39. $\mathrm{Yu} \mathrm{Z,} \mathrm{Ju} \mathrm{Y} \mathrm{and} \mathrm{Liu} \mathrm{H}$ : Antilung cancer effect of glucosamine by suppressing the phosphorylation of FOXO. Mol Med Rep 16 3395-3400, 2017

40. Machado J, Manfredi LH, Silveira WA, Gonçalves DAP, Lustrino D, Zanon NM, Kettelhut IC and Navegantes LC: Calcitonin gene-related peptide inhibits autophagic-lysosomal proteolysis through cAMP/PKA signaling in rat skeletal muscles. Int J Biochem Cell Bio 72: 40-50, 2016
41. Formosa R and Vassallo J: cAMP signalling in the normal and tumorigenic pituitary gland. Mol Cell Endocrinol 392: 37-50, 2014.

42. Haroon N, Aggarwal A, Garg N, Krishnani N, Kumari N and Agarwal V: An unusual case of systemic lupus erythematosus mimic: disseminated gastric signet ring cell carcinoma. Indian J Med Sci 60: 520-522, 2006.

43. Shimoda T, Matsutani T, Yoshida H, Hosone M and Uchida E: A case of gastric cancer associated with systemic lupus erythematosus and nephrotic syndrome. Nihon Shokakibyo Gakkai Zasshi 110: 1797-1803, 2013 (In Japanese).

44. Velusamy T, Palanisamy N, Kalyana-Sundaram S, Sahasrabuddhe AA, Maher CA, Robinson DR, Bahler DW, Cornell TT, Wilson TE, Lim MS, et al: Recurrent reciprocal RNA chimera involving YPEL5 and PPP1CB in chronic lymphocytic leukemia. Proc Natl Acad Sci USA 110: 3035-3040, 2013.

45. Sun D, Zhou M, Kowolik CM, Trisal V, Huang Q, Kernstine KH, Lian F and Shen B: Differential expression patterns of capping protein, protein phosphatase 1 , and casein kinase 1 may serve as diagnostic markers for malignant melanoma. Melanoma Res 21: 335-343, 2011.

46. Sander B, Xu W, Eilers M, Popov N and Lorenz S: A conformational switch regulates the ubiquitin ligase HUWE1. Elife 6: e21036, 2017

47. Ma W, Zhao P, Zang L, Zhang K, Liao H and Hu Z: Tumour suppressive function of HUWE1 in thyroid cancer. J Biosci 41: 395-405, 2016.

48. Yang Z, Zhuang L, Szatmary P, Wen L, Sun H, Lu Y, Xu Q and Chen X: Upregulation of heat shock proteins (HSPA12A, HSP90B1, HSPA4, HSPA5 and HSPA6) in tumour tissues is associated with poor outcomes from HBV-related early-stage hepatocellular carcinoma. Int J Med Sci 12: 256-263, 2015.

49. Park JM, Kim JW and Hahm KB: HSPA4, the 'Evil Chaperone' of the HSP family, delays gastric ulcer healing. Dig Dis Sci 60: 824-826, 2015

c) (i) $\ominus$ This work is licensed under a Creative Commons Attribution-NonCommercial-NoDerivatives 4.0 International (CC BY-NC-ND 4.0) License. 